\title{
Organics settle down
}

\section{Researchers are slowly but surely improving the processing of organic semiconductors, making organic devices more viable.}

The use of organic and molecular materials in devices has traditionally involved a trade-off. In the 'plus' column are the potential for low cost, ease of fabrication, and unique properties such as flexibility and transparency. In the 'minus' column are their fragility and relatively poor conduction. This 'yin and yang' has been grudgingly acknowledged as inevitable. For example, the lack of long-range order that reduces charge mobilities also imparts flexibility: indeed, it seems hard to imagine one without the other. Three papers in this issue, however, demonstrate significant improvements in the conductivity, processing and patterning of organic materials, suggesting that the 'minus' column may be growing shorter and that these trade-offs may not be so inevitable.

\section{"All prerequisites for efficient charge transport in a field- effect transistor are fulfilled."}

On page 674, Dago de Leeuw and colleagues fabricate a field-effect transistor in which the channel between the source and drain electrodes consists of a selfassembled monolayer of liquid-crystalline organic molecules. The molecules contain spacer groups that lead to ordered stacking, and de Leeuw and co-workers have previously reported ${ }^{1}$ that monolayers of these molecules have a mobility that is similar to that of a bulk single crystal, with good performance and repeatability. They also observed that the mobility of this channel did not scale with channel length (whereas the mobility of transistors made from self-assembled monolayers usually decreases dramatically as the length of the channel is increased).

In their latest work de Leeuw and colleagues demonstrate that this unusual scaling behaviour results in part from unusually good conduction characteristics. The injection of charges into the channel does not limit conduction, and neither does the presence of grain boundaries nor the sizes of conducting islands in the channel itself. As a result, the conduction only depends on the channel length when the monolayer is incomplete. The data indicates that, in the words of the authors, "all prerequisites for efficient charge transport in a field-effect transistor are fulfilled." Molecular self-assembled transistors are, therefore, moving beyond the very short channels and low yields of their past.

On page 658 David Vanden Bout and colleagues focus on an organic molecule that self-assembles into nanotubes rather than monolayers. The resulting structure, called a J-aggregate, has attracted attention because of its narrow emission spectrum, high excited-state mobilities and photoconductivity, among other properties. However, using J-aggregates in solid-state devices, for example as light harvesters or energy transporters, will involve immobilizing them on substrates while preserving their structure and optoelectronic properties. To show that this is possible, Vanden Bout and co-workers used polarization-resolved near-field scanning optical microscopy to examine a collection of J-aggregates dispersed onto a substrate in two ways: a traditional spin-coating technique and a drop-flow technique that consisted of simply pouring a solution of J-aggregates onto a substrate, which was then air dried. The microscopy revealed that while spin coating damaged the structures, the drop-flow approach did not. Moreover, the undamaged J-aggregates showed remarkably uniform structure and emission along individual nanotubes, and also among different nanotubes within ensembles. In addition to showing that the structures survive, the data also confirm how well-ordered they really are. Further studies of the transport of electron-hole pairs down the lengths of J-aggregates may aid the development of applications of these structures.

Finally, on page 664, Franco Cacialli and colleagues focus on yet another kind of organic material: poly( $p$-phenylene vinylene), or PPV, a workhorse material that has been investigated for transistors, solar cells and light-emitting devices. Making nanoscale structures out of PPV, however, has been a challenge. This is because it cannot withstand traditional patterning methods such as classical photolithography, which requires the applications of masks and the use of harsh solvents. Researchers have focused instead on 'soft lithography' techniques such as stamping, direct-writing (in which a focused beam of light, electrons or ions creates the pattern required) and approaches based on scanning probes (which rely on interactions between the probe and the sample to alter the surface).

Cacialli and co-workers focus on a probe-based approach that relies on heat to pattern the surface. Like other probe-based approaches, this one offers a relatively low throughput, since patterns are fashioned one at a time, rather than in parallel. But the team make this technique more attractive by greatly increasing its write speed and demonstrating a $28 \mathrm{~nm}$ feature size even though the heat is applied by a wire that is $50 \mu \mathrm{m}$ in diameter. The small feature size is made possible by the small substrate-wire contact area and temperature variations along the height of the polymer film. As Amar Basu and Yogesh Gianchandani point out in an accompanying News \& Views on page 622, the technique is also notable for its wide applicability to other polymers, as well as to materials to which a cross-linker can be added.

\section{As this work progresses, the advantages of using organic materials in the first place remain compelling.}

These three studies suggest that the 'cheap and easy' processing of organic materials will, in fact, take a fair amount of work to be realized (ref. 2 provides links to all the papers we have published about organic materials). But they also show us that as this work progresses, the advantages of using organic materials in the first place remain compelling. After all, it is still the case that, in these reports, the transistor channel was self-assembled, the J-aggregates were self-assembled and poured onto a substrate out of a beaker, and the patterned polymer was spin-coated onto its substrate.

\section{References}

1. de Leeuw, D. M. et al. Nature 455, 956-959 (2008). 2. http://www.nature.com/nnano/focus/organics/index.html 